\title{
Scanning electrochemical microscopy for the investigation of localized degradation processes in coated metals
}

\author{
Yaiza González-García, Juan José Santana+, Jorge González-Guzmán, Javier \\ Izquierdo, Sergio González and Ricardo M. Souto \\ Department of Physical Chemistry, University of La Laguna, E-38207 La Laguna, Tenerife, \\ Canary Islands, Spain
}

\begin{abstract}
$\underline{\text { Abstract }}$
Scanning electrochemical microscopy has been employed to obtain spatially-resolved information regarding surface topology, water uptake and blister formation at intact coatings, as well as the onset and progress of corrosion reactions within coating defects. Topographical lines and maps as well as chronoamperometric plots were measured during operation in the feedback mode. Next, the release of metal ions at the anodic sites and the consumption of oxygen at the cathodic sites developed in holidays could be monitored during operation in the generator-collector mode. Furthermore, the surface topography of defective coatings was imaged by using the redox competition mode.
\end{abstract}

Keywords: Scanning electrochemical microscopy; Paint coatings; Degradation; Blistering; Water uptake.

+ On leave from the Department of Process Engineering, University of Las Palmas de Gran Canaria, Canary Islands, Spain. 


\section{Introduction}

Electrochemistry provides a powerful means of studying interfacial reactions, in particular corrosion transformations. Indeed, a major part of the current knowledge of corrosion processes has been gained from the use of electrochemical methods in the corrosion laboratory. But corrosion reactions are initiated within the range of nanometers and micrometers, and a comprehensive understanding of the mechanistic aspects of corrosion requires the synthesis of data obtained in those scales. Unfortunately conventional electrochemical techniques lack spatial resolution and provide little information on behaviour at sites of corrosion initiation or at defects. Thus, there is need for ancillary techniques with enhanced spatial resolution which can acquire data in real time, and can provide corroborative or other novel evidence of the underlying reaction schemes. The recent advent of a number of local probe techniques is greatly contributing to overcome those limitations when applied to the investigation of corrosion processes in situ. Investigators have explored a wide variety of methods to characterize local electrochemical processes, which range from the design of microelectrochemical cells and setups to the use of scanning probe techniques (the reader is referred to ref. 1 for an authoritative and comprehensive review of these methods as employed in corrosion research).

Microelectrochemical techniques consist basically of scanning a microelectrode over the immersed specimen, either in a static mode (scanning reference electrode technique, SRET) [2-4] or in a vibrating mode (scanning vibrating electrode technique, SVET) [5-8]. In this way either current or potential mapping at the surface of coated metals has been obtained.

An interesting alternative approach has resulted from a variation of local probe microscopies in which a ultramicroelectrode tip (UME) is used as the scanning probe leading to the development of the scanning electrochemical microscope (SECM) [9-11]. A faradaic current is measured at the tip, which serves as a local probe. The current is controlled by diffusion of oxidizable/reducible species used as mediator in the solution, and it depends strongly on the tip-sample separation. Scanning the UME over a defined surface, an image of the surface can be generated. The technique has been successfully applied to several corrosion problems, such as pitting corrosion [12-17], the galvanic corrosion of zinc-iron [18,19], mapping of the surface conductivity of passive layers [20,21], the influence of inhibitors [22,23], the examination of organic coatings on metallic substrates [24-30] and, the galvanic activity at defective coated metals [31,32].

The Scanning Electrochemical Microscope is a nonoptical scanning microscopic instrument capable of imaging highly localized electrical currents associated with charge transfer reactions occurring in the vicinity of surfaces. The SECM tip operates as working microelectrode in an 
electrochemical cell under potentiostatic control. Microelectrode current and position is recorded as the tip is scanned very near a sample at costant $Z$ height. The variations in the faradaic current measured can be related either to local differences in electrochemical reactivity at the sample or changes in the distance between the tip and the surface of the sample depending of SECM operation mode.

Three different operation modes have been found to be applicable for the local investigation of corrosion reactions. Firstly, the "Feedback Mode": The SECM response arises from the perturbation of the tip current when the tip is brought within a few tip diameters of a surface. An electrochemical mediator is employed. Secondly, the "Generation/Collection Mode": The tip current is used to monitor the flux of electroactive species from or to the substrate. The potentiometric tip is a passive sensor. Finally, in the "Redox Competition Mode" the soluble electrochemical mediator reacts at both the ultramicroelectrode and the substrate, and a competition is established between the two active surfaces for the redox species. For a flat surface, the current measured at the tip is then smaller over a reacting site that over the surrounding insulating surface.

\section{Scanning Electrochemical Microscopy}

\subsection{The technique}

The SECM instrument consists of a combination of electrochemical components and some components of the SPMs. A diagram with the main components of the scanning electrochemical microscope is shown in Figure 1. It consists of four major parts [33]:

The tip movement and position controller, which is the SPM component.

$>$ The electrochemical cell, which is formed by the tip, the counter electrode, the reference electrode and the substrate that occasionally, can act as second working electrode.

> The bipotentiostat, which constitutes the SECM electrochemical setup together with the electrochemical cell.

$>$ Computer, interface and display system.

\subsection{Principles of the SECM}

The SECM is a technique in which current flows through a microelectrode immersed in an electrolytic solution and situated close to a substrate. The substrate can either be a conductive, semiconductive or insulating material. The microelectrode and the substrate form part of an 
electrochemical cell which is also constituted by reference and auxiliary electrodes, and sometimes by the substrate as a second working electrode (Figure 1).

Prior to explaining the operation and the response of the SECM, it is necessary to understand the behaviour of the microelectrode inside an electrochemical cell. When the electrode surface is comparable to or smaller than the diffusion layer thickness, the diffusion process becomes dependent on the size and geometry of the electrode [34-38]. The diffusive regime is the characteristic feature of microelectrodes in this case, and for a typical planar microdisk electrode, mass transport is controlled totally by hemispherical diffusion between the solution bulk and the electrode surface (see Figure 2A).

Let's consider the microelectrode is immersed in a solution containing an electrolyte and a reducible species, $O$. When a potential sufficiently negative to the standard potential of the reduction reaction (1), is applied to the microelectrode, the reduction of the species $O$ occurs at the UME surface, and a cathodic current flows according to:

$$
O+n e^{-} \leftrightarrow R
$$

If this reaction is kinetically controlled by the diffusion of $O$ from the bulk of the solution to the electrode surface, the current decays due to the formation of a diffusion layer of $O$ around the electrode, and attains rapidly a steady-state value given by

$$
i_{\infty}=4 n F D c a
$$

where $F$, is the Faraday constant; $a$, the microelectrode radius; $D$, the diffusion coefficient of the reducible species; and, $c$, its concentration. A steady-state current results from the constant flux of $O$ to the electrode surface due to an expanding hemispherical diffusion layer around the microelectrode.

In SECM, the proximity of the tip to the substrate is the perturbation in the measurement that constitutes the SECM response [39]. If the UME is brought to the vicinity of an insulating substrate, the steady-state current that flows through the tip, $i_{\text {hind }}$, tends to be smaller than $i_{\infty}$ (Figure $2 \mathrm{~B}$ ). This is a result of the insulating substrate partially blocking the diffusion of $O$ towards the tip [40]. The current at the tip becomes smaller when the tip is closer to the substrate, and tends to zero when the distance between tip and substrate, $d$, approaches zero. This effect is known as negative feedback.

In contrast, if the tip is close to a conductive substrate at which the oxidation reaction (3) can occur, a flux of $O$ from the substrate to the tip occurs, in addition to some flux from the bulk solution towards the tip. 


$$
R \leftrightarrow O+n e^{-}
$$

This effect leads to an enhancement of the current at the tip, $i_{t i p}$, which is higher than $i_{\infty}$ (Figure 2C). Then the flux of $O$ from the substrate to the tip causes an increase of $i_{\text {tip }}$ as $d$ decreases. In this case, when the tip-substrate distance approaches zero, the $i_{\text {tip }}$ current tends to infinite, and the oxidation of $R$ on the substrate is diffusion-controlled. This effect is named positive feedback. The tip can be seen as both the generator of the signal sensing the substrate (the flux of the reduced species, $R$ ), and the detector (of the flux of $O$ from the substrate).

\subsection{Operation modes}

The SECM can be employed to image the surfaces of different types of substrates, both insulators and conductors, immersed in solutions. In this case, the SECM operates in the feedback mode. Then, it is necessary an oxidizable/reducible species in the solution which is known as redox mediator (cf. Figure 3). Generally redox couples involving a fast, usually one-electron, heterogeneous reaction at the tip are chosen as electrochemical mediators. In this mode, the SECM tip is rastered in the $X-Y$ plane of the surface of the sample inside an electrochemical cell. The faradaic current measured at the tip is controlled by diffusion of the mediator, and depends strongly on the tip-sample separation. The variations in the measured current are related to changes in the tipsample distance and therefore, they are related to the topography of the sample. In the case of an insulator surface such as a non-conductive polymer coating, the diffusion of the redox mediator from the bulk solution to the tip surface becomes progressively hindered as the tip approaches the surface (i.e., negative feedback effect). The diffusion-hindered current is thus directly related to the tipsubstrate distance $d$.

Topography imaging is often determined with the SECM operating in the constant height mode. In this way, the tip is lowered to a fixed distance from the substrate and then the surface is scanned. In this case, the currents measured at the tip can be rapidly converted to a height or distance scale. The resolution of the SECM in topographic measurements on a surface strongly depends on the tip radius, $a$.

The SECM can be used as an electrochemical tool to study electrode processes and coupled homogeneous reactions by using the generation-collection operating modes. In the substrate generator - tip collector mode, the tip current is used to monitor the flux of electroactive species from the substrate. This configuration is applied in some cases to determine concentration profiles 
above a substrate electrode. On the other hand, the SECM can be used in the tip generator - substrate collector mode, in which the substrate monitors the flux generated from the tip. This mode is useful in studies of homogeneous reactions that occur in the tip-substrate gap. Figure 3 depicts the basic principle of the SECM used in either the substrate generator - tip collector mode for an active site on the otherwise insulating surface.

A new situation occurs when the same redox species can react at both the tip and the substrate, thus originating a competition for the species between them. In the redox competition mode, the substrate monitors smaller currents than in the corresponding feedback mode due to the partial consumption of the redox mediator at the substrate, this effect becoming increasingly noticeable at smaller tip-substrate distances (see Fig. 3).

\subsection{Experimental procedure}

Imaging with the SECM was always conducted in the constant height mode, in either the surface image or the SECM electrochemical modes. The choice of the operation mode was based on the source of the electrochemical species participating in the redox processes responsible for the faradaic currents sensed at the tip. The redox mediators used, and the potential values employed to attain their diffusion-limited regimes, are listed in Table 1.

The approach of the tip from the bulk solution to the surface of the sample was accomplished by slowly approaching the tip to the surface, and simultaneously recording the approach curve as the measured current at the microelectrode versus $Z$ displacement (cf. Figure 2B). It was considered that the tip reached the insulating surface when the reduction of the diffusion-limited current at the microelectrode reached a chosen fraction of the value in the bulk solution. The tip actually never touched the surface of the sample.

\section{Results and discussion}

The principles of these methods will be illustrated by considering some applications of SECM to the study of localized corrosion processes:

\subsection{SECM operating in the Feedback mode: Observation of the early stages of degradation and} blistering formation processes of a coated metal. 
Evolution of the coated metal during its immersion in aqueous electrolyte solution has been observed in situ by SECM technique. The current measured at the tip was controlled by diffusion of a mediator (ferrocene-methanol, molecular oxygen), and it depended on the tip-sample separation. In this way, both the uptake of water by the polymeric film and the initiation of swelling at the metalpolymer interface could be investigated.

The permeation of water into the polymeric matrix should result in an increase of its volume, and the surface thus progressing into the solution phase. In the SECM system, this process should originate the surface of the substrate effectively approach the tip when rastered at the same height continuously over the substrate. Line scans were thus recorded as a function of immersion time in the electrolyte to detect such a trend in the experimental system. Figure 4 displays graphs produced by selecting typical line-scans corresponding to different exposure periods in various test solutions. Due to the unavoidable tilt of the samples, all the line-scans exhibit small changes in the values of the current measured at the tip whereas the surface is scanned. The tilt is clearly evidenced specially for the sample immersed in $0.01 \mathrm{M} \mathrm{KCl}$ solution (see Figure 4B). This tilt effect does not originate from the processes that occur inside the electrochemical cell during an experiment, but from the geometry of the SECM cell. Thus, for a given experiment, its value is time-independent. Nevertheless, the tip measurement will be more sensitive to variations in the tip-sample distance at that part of the surface which is more proximate, thus lines are not parallel but separate at the left of the figure where the substrate is closer to the tip. Conversely, such effect is almost completely absent for the line-scans depicted in Figure 4A taken in $0.1 \mathrm{M} \mathrm{K}_{2} \mathrm{SO}_{4}$ for which the initial tilt was smaller.

The current values measured in the various line-scans are always observed to be smaller than those obtained from the previous one during the first 5 hours exposure, but they tend to superimpose for longer exposures. SECM is thus sensitive to changes in the absolute position of the surface of the coated metal in relation to the position of the tip, and can be used to monitor water permeation through the polymeric matrix.

This effect could be further investigated by performing another kind of experiment. In this case, the tip was not scanned over the coated surface. That is, the UME was maintained above a random point of the surface. As the tip was maintained in the near-field range of the system, variations in the current flowing at the tip during this experiment resulted from changes in the effective tip-substrate distance with time. The corresponding chronoamperometric curves measured for CCS samples immersed either in $0.1 \mathrm{M} \mathrm{K}_{2} \mathrm{SO}_{4}$ or in $0.1 \mathrm{KCl}$ are plotted in Figure 5. In this particular case, the two current traces exhibit a similar rate of change. They depict an initial decrease in current immediately after the immersion of the sample, which levels off after approximately 1000 seconds. The shape of the current transient agrees well with the most recognized model of water 
uptake trend in organic coatings [41-43]. Indeed, from the inspection of figure 5 the initial decrease in current can be related to a homogeneous water diffusion in the coating (phase I), whereas the subsequent levelling off corresponds to the saturation of the polymeric matrix (phase II). The final increase of water content responsible for the coating breakdown (phase III) was not observed in our experiment, as significantly longer exposures would be necessary. The natural evaporation of the aqueous solution in the tiny cell employed imposed a severe constraint to the exposure times currently available in our system. To overcome this limitation in the future, new designs for the microelectrochemical cell are under way, which are aimed to guarantee constant concentration in the confined volume of electrolyte during longer exposures.

A separate process is also deduced from the inspection of the line-scans measured in $0.1 \mathrm{M}$ $\mathrm{KCl}$ solution as depicted in Figure 4C. That is, in addition to the water-uptake process, heterogeneous roughening of the polymer surface due to swelling of the organic coating also occurred. This effect is clearly seen when surface maps are performed with the SECM. Whereas rather smooth and featureless surfaces were observed at all times for the samples immersed in chloride-free solutions and even in a rather dilute chloride solution such as $0.01 \mathrm{M} \mathrm{KCl}$, localized areas in the maps where significantly reduced tip currents are monitored could be detected in $0.1 \mathrm{M}$ KCl. As schematically illustrated in Figure 6, reduced currents are measured at the microelectrode, though scanning at constant height over the substrate, where hill-shaped topographic features occur because they hinder the diffusion regime of the redox mediator to the tip.

The chloride-induced swelling of the coating at a microscopic level can be observed in Figure 7 A-B for two different metal-polymer systems. The development of bulges was attributed to the development of microscopic blisters even at very short exposure times for a variety of metal-polymer systems $[24,26-28,30]$. Local swelling was not seen during immersion in the sulphate solution by SECM within this time scale cf. Figure 7C.

Additionally, the advancement towards the tip of an individual feature formed on the polyurethane coating can also be investigated. The distance between the summit of a selected blister and the microelectrode was determined from a series of SECM images measured sequentially for a randomly chosen area of the surface. The current measured at the tip could then be correlated to the tip-substrate distance by using the approach curve measured over the polyurethane-coated sample, which is depicted in Figure 8. The progression towards the microelectrode of the blister detected in Figure 7B during the exposure of the coated sample to the chloride-containing solution, was followed during 5 hours. Figure 9 exhibits the evolution of the distance between the tip and the blister during that interval of time. Within the first hour of immersion, the coating retracted locally from the microelectrode for a maximum distance of $2.7 \mu \mathrm{m}$ (Zone I). The coating actually shrinked 
during the process, an indication that some components lixiviated from the polymer matrix simultaneously to water uptake from the electrolytic solution. At longer exposures, roughening of the surface occurs with the formation of bulges, and a steady trend growth of the blister is maintained for the rest of the experiment (Zone II). That is, the coating advanced locally towards the solution phase for about $13 \mu \mathrm{m}$ during the next 4 hours of exposure. This feature was induced by the presence of chloride ions in the solution phase.

\subsection{SECM operating in the Generation/Collection and Redox Competition modes: Investigation of} degradation processes at defective coated metals.

Operation of the SECM in the sample generation-tip collection mode (TG-SC) allows to detect the occurrence of localized corrosion processes and to measure local variations in the extent of metal corrosion from the on line monitoring of species participating in the corrosion processes at defective points of the coating [29,31]. Corrosion at the holiday in the organic coating occurred when the underlying metal substrate was exposed to the aerated electrolytic solution resulting in the release of Fe(II) ions in the anodic half-cell reaction. The cathodic process consumed dissolved oxygen from the solution phase.

The progress of corrosion at the holiday in the organic coating could be followed as a function of time by SECM. Corrosion occurred when the underlying metal substrate was exposed to the aerated electrolytic solution resulting in the release of Fe(II) ions in the anodic half-cell reaction. The cathodic process consumed dissolved oxygen from the solution phase. The ferrous ions were detected at the UME through their oxidation to Fe(III) by setting the tip potential at $+0.60 \mathrm{~V}$, as depicted in Figure 10A. Analogously, the electroreduction of soluble oxygen was monitored when the UME was polarized at $-0.70 \mathrm{~V}$ (cf. Figure 10B). In this way, changes in the local concentration of both ferrous ions and oxygen were recorded by scanning the microelectrode over the holiday, when the appropriate potential values were set at the tip. Therefore, the occurrence of the corrosion reaction at the defect in the coating can be observed as both a local increase in the concentration of ferrous species and a decrease in oxygen concentration.

Figure 11 shows a series of line scans alternately measured to record the concentration profiles for ferrous ions and soluble oxygen over the holiday during immersion in $0.1 \mathrm{M} \mathrm{KCl}$. Degradation of the metal occurred inside the holiday and the release of $\mathrm{OH}^{-}$ions in the cathodic reaction modified the composition of the electrolyte to cause the precipitation of iron corrosion products, which eventually led to blockage of the corroding defect at sufficiently long exposure times. 
These facts are better observed from the images given in Figure 12. These maps are built by representing the spatial variation of the currents measured at the tip for the electroreduction of soluble oxygen in the electrolyte phase within the near field range of the technique. Two different electrolytic environments are considered, $0.1 \mathrm{M} \mathrm{Na}_{2} \mathrm{~B}_{4} \mathrm{O}_{7}$ and $\mathrm{Na}_{2} \mathrm{SO}_{4}$, in which the metal exposed in the holiday is either passivated or undergoes corrosion, respectively. In the former case, the holiday is imaged in the corresponding map as the region of the sample with enhanced currents for oxygen electroreduction (see Figure 12A). The image results exclusively from the SECM operating in the feedback mode as it follows. Hindered currents were measured when the tip scanned over the coated surface, whereas bigger currents were measured when passing above the holiday, because diffusion of the redox species was greatly facilitated compared to the surrounding coated areas due to the availability of oxygen in the electrolyte filling the holiday. It must be noticed that the dimensions of the holiday are significantly bigger than those of the microelectrode (namely 200 and $10 \mu \mathrm{m}$, respectively), and the metal surface lies at a lower height than the surrounding coated areas. This case corresponds to the topographic imaging situation depicted in Figure 3, in which greater currents are detected over the holiday.

The progress of corrosion reactions produces the combined release of metal ions from the anodic sites and the corresponding consumption of oxygen at the cathodic locations developed inside the holiday. The depletion of oxygen within the holiday progressively compensates for the enhanced supply of this species from the bulk electrolyte due to the geometry of the system. For sufficiently long exposures, this effect may eventually lead to the condition where lower currents for oxygen electroreduction are measured at the tip when passing over the holiday. This is the situation monitored in figure $12 \mathrm{~B}$ for the sample exposed to $0.1 \mathrm{M} \mathrm{Na}_{2} \mathrm{SO}_{4}$ during 7 hours. The holiday is located in the region where the microelectrode experiences smaller faradaic currents as result of the redox competition operation.

It should also be noticed that the information obtained with the SECM is not restricted to the holiday in this case, but the technique is sensitive to the topology of the insulating coated areas as well. The electroreduction of soluble oxygen at the tip allowed to image the topology of the coating as to view a scratch extending from left to right in Figure 12A. It must be noted that the underlying metal has not been exposed this time and thus all the surface around the holiday is electrochemically insulating. This feature and the remaining surface have been imaged by recording the current due to hindered diffusion of oxygen at the tip (i.e. negative feedback).

\section{Summary}


It has been demonstrated that great accuracy mapping of the processes and reactions responsible for the degradation of coated metals at the open-circuit potential can be performed using the scanning electrochemical microscope. This originates from the use of the technique under various operation modes, and resolution is mainly determined by the size of the microelectrode.

Operation of the SECM in the feedback mode is sensitive to changes in the absolute position of the surface of the coated metal in relation to the position of the tip, and can be used to monitor the water uptake process that occurs in the coating film during its immersion in an aqueous environment. Water permeates into the polymeric matrix resulting in an increase of its volume, and the surface thus progressing into the solution phase. Such determination can be made simultaneously to the detection of blister formation in the case of chloride-containing solution, which resulted from the local accumulation of the electrolyte at the metal/coating interface.

Early degradation of coated systems induced by chloride species is imaged by SECM under feedback operation. Swelling of the coating gives rise to small bulges observed at very short immersion times in $0.1 \mathrm{M} \mathrm{KCl}$ aqueous solution. This local swelling was attributed to the development of microscopic blisters and to the early stages of degradation processes in the coating. The surface of the coated metals remains flat and there is no indication of bulge formation when the samples are exposed to either a dilute chloride solution, or to a chloride-free solution. The metallic substrate and the nature of polymeric coating do not significantly seem to influence the processes occurring during the early stages of coating degradation.

Finally, SECM can also be employed to monitor corrosion processes occurring at defective coated metals. Painted samples in which a holiday was produced prior to immersion in the test electrolyte were investigated with this technique. The release of Fe(II) ionic species into the solution phase from local anodic sites, as well as the consumption of dissolved oxygen at the corresponding cathodic locations was successfully monitored. The corrosion of the exposed metal substrate in the holiday was observed, as well as the blockage of the corrosion process due to product precipitation at longer exposure times. Surface imaging can be achieved through the electroreduction of soluble oxygen at the tip, though in this case the onset of a redox competition process between the cathodic microelectrodes within the active site and the tip must be considered to adequately describe the observations.

\section{Acknowledgement:}

We are grateful to the Ministerio de Educación y Ciencia (Madrid, Spain) within the framework of Project CTQ2005-06446/BQU, under which the present work was carried out. A 
Doctoral Grant to Y.G.-G. by the University of La Laguna and Cajacanarias (Tenerife, Spain) is gratefully acknowledged.

\section{References:}

1. P. Marcus and F. Mansfeld, Eds., Analytical Methods in Corrosion Science and Engineering; CRC Press, Boca Raton FL, 2006.

2. I.M. Zin, R.L. Howard, S.J. Badger, J.D. Scantlebury and S.B. Lyon, Progress in Organic Coatings 33 (1998) 203.

3. F.J. Maile, T. Schauer and C.D. Eisenbach, Progress in Organic Coatings 38 (2000) 117.

4. M. Khobaib, A. Rensi, T. Matakis and M.S. Donley, Progress in Organic Coatings 41 (2001) 266.

5. H.S. Isaacs, A.J. Aldykiewicz Jr., D. Thierry and T.C. Simpson, Corrosion 52 (1996) 163.

6. J. He, V.J. Gelling, D.E. Tallman and G.P. Bierwagen, Journal of the Electrochemical Society 147 (2000) 3661.

7. S. Böhm, H.N. McMurray, S.M. Powell and D.A. Worsley, Electrochimica Acta 45 (2000) 2165.

8. D.A. Worsley, D. Williams and J.S.G. Ling, Corrosion Science 43 (2001) 2335.

9. A.J. Bard, F.-R. Fan and M. Mirkin, in Physical Electrochemistry: Principles, Methods, and Applications; I. Rubinstein, Ed.; Marcel Dekker, New York, 1995, p. 209.

10. A.J. Bard and M.V. Mirkin, Eds., Scanning Electrochemical Microscopy; Marcel Dekker, New York, 2001.

11. B.R. Horrocks, in Encyclopedia of Electrochemistry; Vol. 3, A.J. Bard, M. Stratmann and P.R. Unwin, Eds.; Wiley-VCH, Weinheim, 2003, p. 444.

12. D.O. Wipf, Colloid Surface A: Physicochemical and Engineering Aspects 93 (1994) 251.

13. T.E. Lister and P.J. Pinhero, Electrochimica Acta 48 (2003) 2371.

14. Y. González-García, G.T. Burstein, S. González and R.M. Souto, Electrochemistry Communications 6 (2004) 637.

15. C. Gabrielli, S. Joiret, M. Keddam, H. Perrot, N. Portail, P. Rousseau and V. Vivier, Journal of the Electrochemical Society 153 (2006) B68.

16. A. Davoodi, J. Pan, C. Leygraf and S. Norgren, Electrochimica Acta 52 (2007) 7697.

17. K. Eckhard, H. Shin, B. Mizaikoff, W. Schuhmann and C. Kranz, Electrochemistry Communications 9 (2007) 1793. 
18. A.C. Bastos, A.M. Simões, S. González, Y. González-García and R.M. Souto, Electrochemistry Communications 6 (2004) 1212.

19. A.C. Bastos, A.M. Simões, M.G. Ferreira, Y. González-García, S. González and R.M. Souto, Corrosion Science 49 (2007) 726.

20. J.C. Seegmiller and D. Buttry, Journal of the Electrochemical Society 150 (2003) B413.

21. M.A. Malik and P.J. Kulesza, Analytical Chemistry 79 (2007) 3996.

22. Mansikkamaki, K.; Ahonen, P.; Fabricius, G.; Murtomaki, L.; Kontturi, K. Journal of the Electrochemical Society 152, (2005) B12.

23. K. Mansikkamaki, U. Haapanen, C. Johans, K. Kontturi and M. Valden, Journal of the Electrochemical Society 153 (2006) B311.

24. R.M. Souto, Y. González-García, S. González and G.T. Burstein, Corrosion Science 46 (2004) 2621.

25. A.C. Bastos, A.M. Simões, S. González, Y. González-García and R.M. Souto, Progress in Organic Coatings 53 (2005) 177.

26. R.M. Souto, Y. González-García and S. González, Corrosion Science 50 (2008) 1637.

27. R.M. Souto, Y. González-García and S. González, Progress in Organic Coatings 65 (2009) 435.

28. R.M. Souto, Y. González-García and S. González, Electroanalysis (2009), in press, ref. élan_200900232.

29. R.M. Souto, Y. González-García, S. González and G.T. Burstein, Electroanalysis (2009), in press, ref.elan_200900262.

30. R.M. Souto, Y. González-García and S. González, submitted to Corrosion Science (2009), in press, ref. CORSCI-S-09-00465.

31. Y. González-García, S. González and R.M. Souto, Corrosion Science 47 (2005) 3312.

32. A.M. Simões, D. Battocchi, D.E. Tallman and G.P. Bierwagen, Corrosion Science 49 (2007) 3838.

33. J. Kwak and A.J. Bard, Analytical Chemistry 61 (1989) 1794.

34. P. Delahay, New Instrumental Methods in Electrochemistry; Interscience, New York, 1954.

35. R.M. Wightman and D.O. Wipf, in Electroanalytical Chemistry; Vol. 15, A.J. Bard, Ed.; Marcel Dekker, New York, 1989.

36. A.M. Bond, K.B. Oldham and C.G. Zoski, Analytica Chimica Acta 216 (1989) 177.

37. J. Heinze, Angewandte Chemie 103 (1991) 175. 
38. M. Fleischmann, S. Pons and D.R. Rolison, in Ultramicroelectrodes; P.P. Schmidt, Ed.; Datatech System Inc., Morganton, 1987.

39. A.J. Bard, F-R.F. Fan, J. Kwak and O. Lev, Analytical Chemistry, 61 (1989) 132.

40. A.J. Bard, G. Denuault, C. Lee, D. Mandler and D.O. Wipf, Accounts of Chemical Research 23 (1990) 357.

41. F. Deflorian, L. Fedrizzi, S. Rossi and P.L. Bonora, Electrochimica Acta 44 (1999) 4243.

42. S.A. Lindqvist, Corrosion 41 (1985) 69.

43. F. Deflorian and L. Fedrizzi, Journal of Adhesion Science and Technology 13 (1999) 629.

Table 1 - Electrochemical mediators used in the investigation.

\begin{tabular}{|c|c|c|c|}
\hline Mediator & Concentration & Redox couple & $\begin{array}{c}\text { Potential, V } \\
\text { vs. Ag/AgCl/KCl } \mathrm{Kat}\end{array}$ \\
\hline Ferrocene-methanol & $0.5 \mathrm{mM}$ & Ferrocene/Ferrocinium & +0.5 \\
\hline $\mathrm{FeSO}_{4}\left(\mathrm{NH}_{4}\right)_{2} \mathrm{SO}_{4}$ & $5-7 \mathrm{mM}$ & $\mathrm{Fe}^{2+} / \mathrm{Fe}^{3+}$ & +0.6 \\
\hline $\mathrm{K}_{4}\left[\mathrm{Fe}(\mathrm{CN})_{6}\right]$ & $1 \mathrm{mM}$ & $\mathrm{Fe}\left(\mathrm{CN}_{6}\right)^{4-} / \mathrm{Fe}\left(\mathrm{CN}_{6}\right)^{3-}$ & +0.4 \\
\hline Dissolved oxygen & - & $\mathrm{O}_{2} / \mathrm{OH}^{-}$ & -0.7 \\
\hline
\end{tabular}

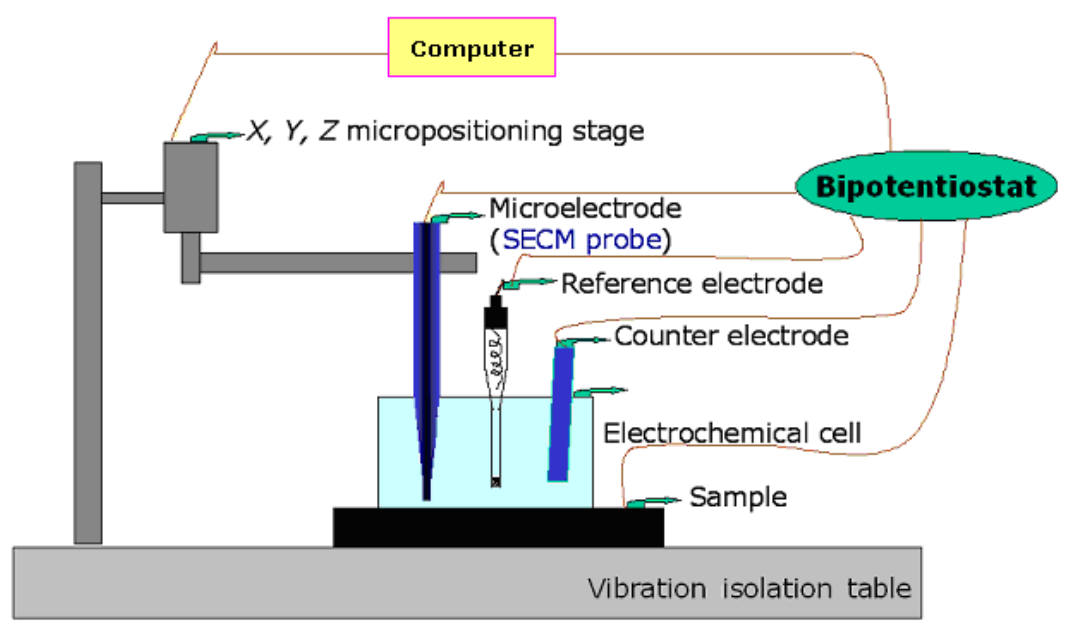

Figure 1:

Schematic representation of the SECM system. 
(A)

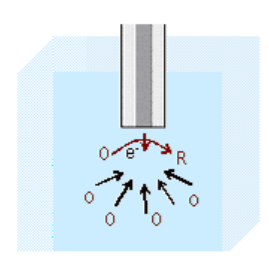

(B)

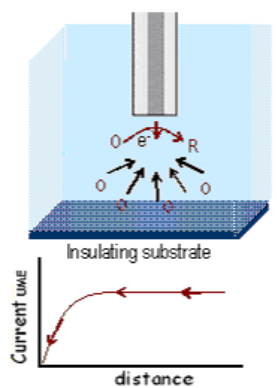

(C)

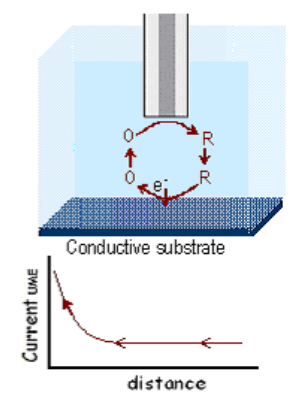

Figure 2:

Basic principle of SECM: (A) Geometry and diffusion field for a microelectrode. (B) Negative feedback: the tip is placed near an insulating substrate which hinders the diffusion of species $O$. (C) Positive feedback: the tip is located near a conductive substrate where the oxidation of species $R$ occurs.

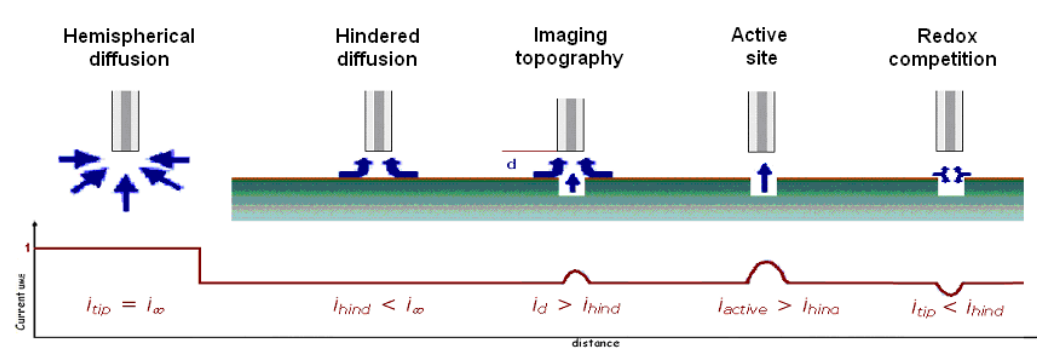

Figure 3:

Operation modes of a SECM and corresponding current responses, expressed as a normalized quantity. Arrows represent the flow of the electroactive species to the SECM tip.

(A)

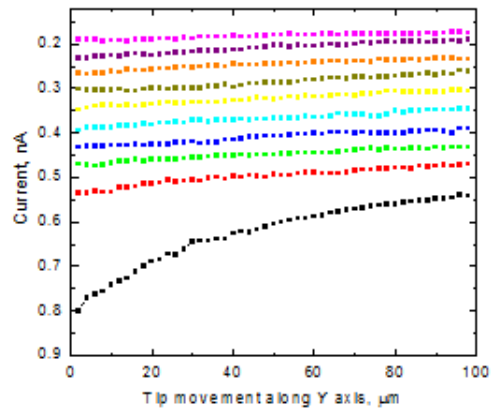

(B)

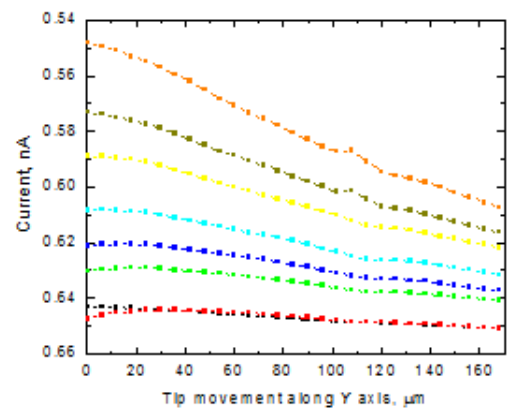

(C)

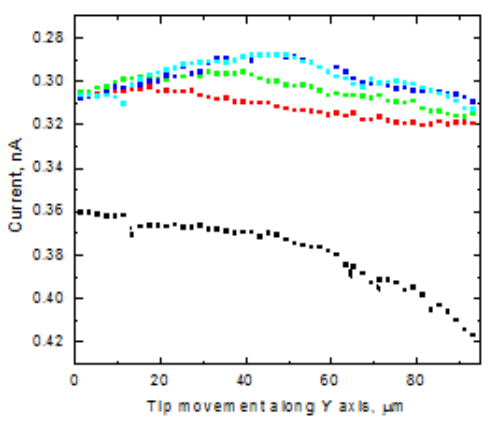

Figure 4:

Line scans measured along $Y$ direction during the exposure of galfan CCS to: (A) $0.1 \mathrm{M} \mathrm{K}_{2} \mathrm{SO}_{4}$

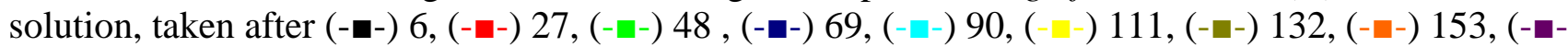
) 174, and (-ø-) 195 min of immersion in the test solution. (B) $0.01 \mathrm{M} \mathrm{KCl}$ solution, taken after (-ø-)

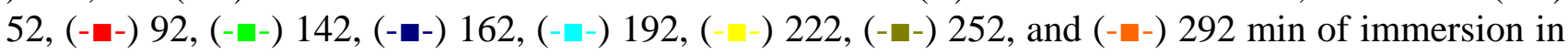
the test solution. And, (C) $0.1 \mathrm{M} \mathrm{KCl}$ solution, taken after (-m-) 6, (-口-) 21, (-口-) 48, (-m-) 64, and (-) $85 \mathrm{~min}$ of immersion in the test solution. Scan rate: $20 \mu \mathrm{m} \mathrm{s}^{-1}$. Redox mediator: ferrocenemethanol. Tip potential: $+0.50 \mathrm{~V}$ vs. $\mathrm{Ag} / \mathrm{AgCl} / \mathrm{KCl}$ (saturated) reference electrode. Tip diameter: 10 $\mu \mathrm{m}$. Tip-substrate distance: $10 \mu \mathrm{m}$. 


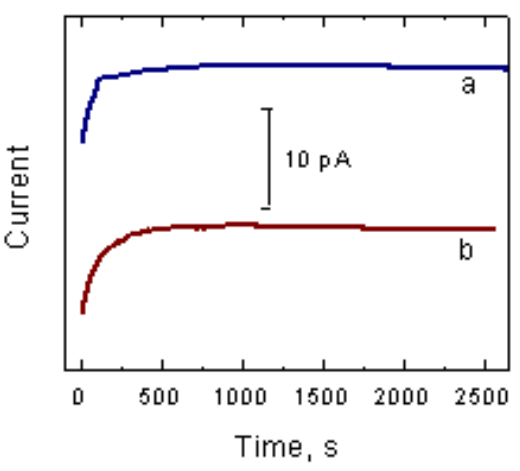

Figure 5:

Chronoamperometric curves measured at the SECM tip when situated at $10 \mu \mathrm{m}$ from the surface of galfan CCS samples immersed in: (a) $0.1 \mathrm{M} \mathrm{K}_{2} \mathrm{SO}_{4}$, and (b) $0.1 \mathrm{M} \mathrm{KCl}$ solution. Redox mediator: ferrocene-methanol. Tip potential: $+0.50 \mathrm{~V}$ vs. $\mathrm{Ag} / \mathrm{AgCl} / \mathrm{KCl}$ (saturated) reference electrode. Tip diameter: $10 \mu \mathrm{m}$. Tip-substrate distance: $10 \mu \mathrm{m}$.

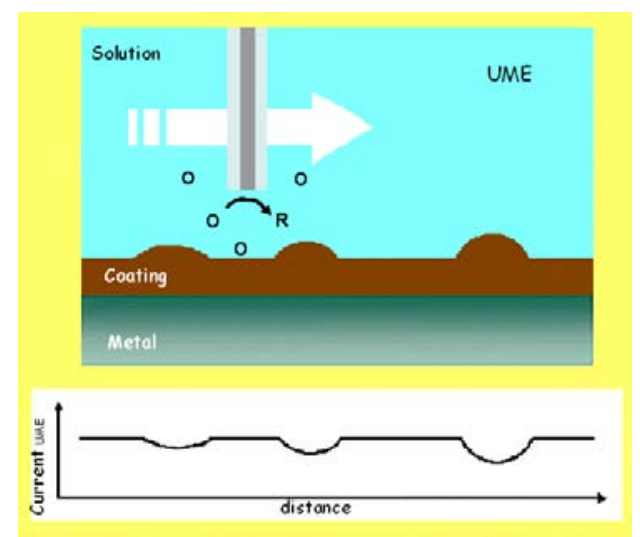

Figure 6:

Schematic representation of the SECM tip operating in the feedback mode for the topographic imaging of a rough insulating surface such as an intact paint coating.

(A)

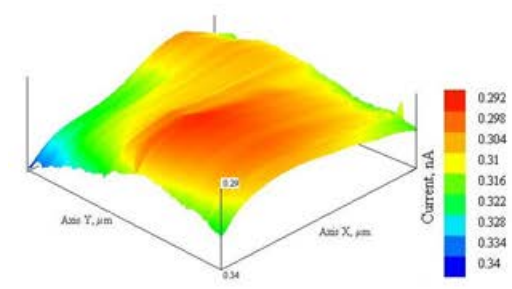

(B)

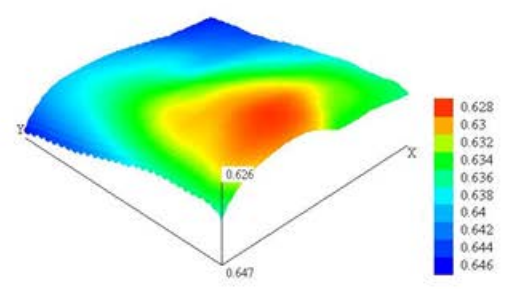

(C)

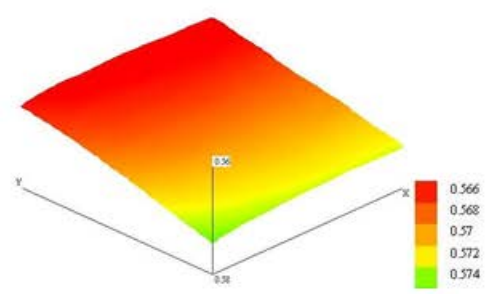

Figure 7:

SECM images of three metal-coating systems: (A) Galfan coil coated steel after 42min immersion in $0.1 \mathrm{M} \mathrm{KCl}$ solution; the image represents $100 \mu \mathrm{m} \times 100 \mu \mathrm{m}$ in $X$ and $Y$ directions; scan rate: $20 \mu \mathrm{m}$ $\mathrm{s}^{-1}$. (B) Polyurethane-coated carbon steel after 142 min immersion; the image represents $200 \mu \mathrm{m} \mathrm{x}$ $200 \mu \mathrm{m}$ in $X$ and $Y$ directions; scan rate: $30 \mu \mathrm{m} \mathrm{s}^{-1}$. (C) Galfan coil coated steel after $6 \mathrm{~h}$ immersion in $0.1 \mathrm{M} \mathrm{K}_{2} \mathrm{SO}_{4}$ solution; the image represents $100 \mu \mathrm{m} \times 100 \mu \mathrm{m}$ in $X$ and $Y$ directions; scan rate: 25 $\mu \mathrm{m} \mathrm{s}^{-1}$. Values of $Z$ axis: Current, $\mathrm{nA}$. Redox mediator: ferrocene-methanol. Tip potential: $+0.50 \mathrm{~V}$ vs. Ag/AgCl/KCl (saturated) reference electrode. Tip diameter: $10 \mu \mathrm{m}$. Tip-substrate distance: 10 $\mu \mathrm{m}$. 


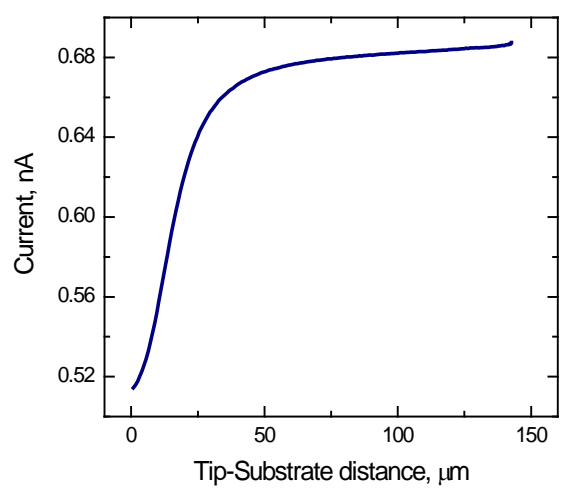

Figure 8:

Approach curve measured at the SECM-tip in $0.1 \mathrm{M} \mathrm{KCl}+0.5 \mathrm{mM}$ ferrocene-methanol solution shortly after the polyurethane-coated carbon steel sample was exposed to the solution. Redox mediator: ferrocene-methanol. Tip potential: $+0.50 \mathrm{~V}$ vs. $\mathrm{Ag} / \mathrm{AgCl} / \mathrm{KCl}$ (saturated) reference electrode. The tip was regarded to have reached the surface when the current measured at the tip decreased to a $75 \%$ of the steady-state value in the bulk of the electrolyte. Tip diameter: $10 \mu \mathrm{m}$.

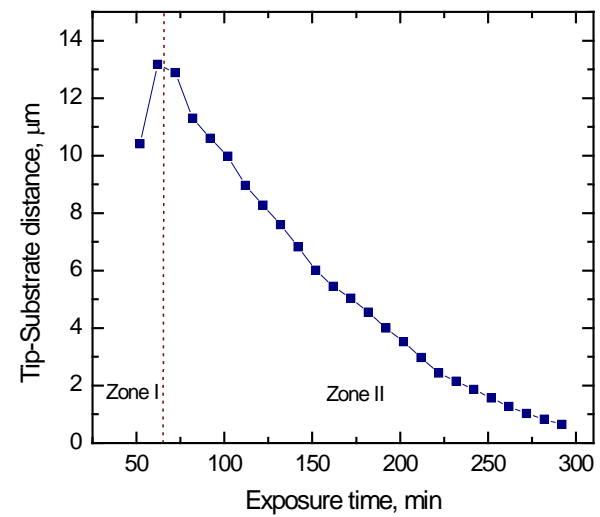

Figure 9:

Distance between the tip and the summit of a blister developed on a polyurethane-coated CCS specimen immersed in $0.1 \mathrm{M} \mathrm{KCl}$ as a function of exposure time. The swelling under consideration is the same depicted in Figure 7B.

(A)

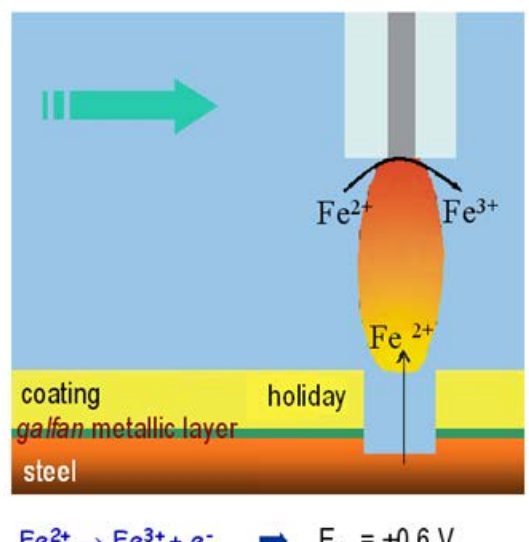

$\mathrm{Fe}^{2+} \rightarrow \mathrm{Fe}^{3+}+e^{-} \Rightarrow \mathrm{E}_{\text {tip }}=+0.6 \mathrm{~V}$
(B)|

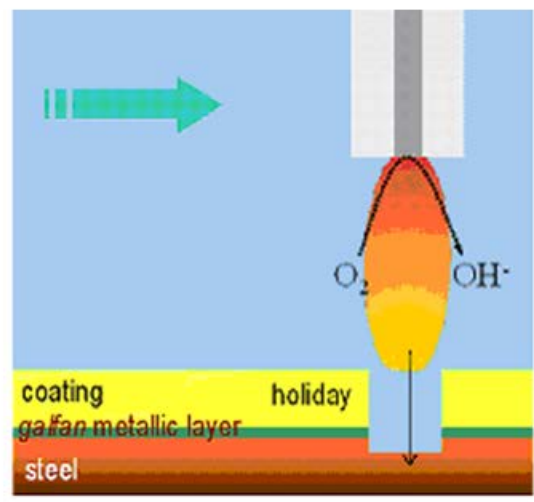

$\mathrm{O}_{2}+2 \mathrm{H}_{2} \mathrm{O}+4 \mathrm{e}^{-} \rightarrow 4 \mathrm{OH}^{+} \Rightarrow \mathrm{E}_{0 \mathrm{jp}}=-0.7 \mathrm{~V}$

Figure 10:

Schematic representations of the SECM tip operating in the generator-collector mode for the imaging of the electrochemical activity occurring at a holiday operated in painted galvanized steel. 
(A)

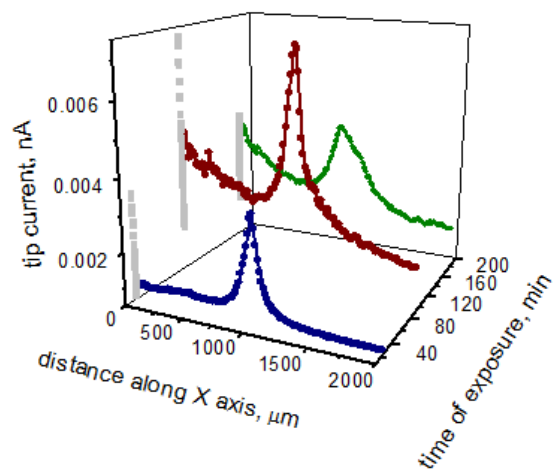

(B)

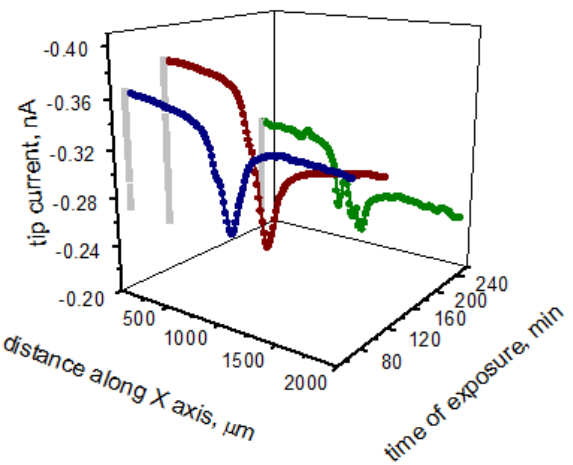

Figure 11:

Line scans across the holiday operated in polyurethane coated steel after immersion in $0.1 \mathrm{M} \mathrm{KCl}$. The tip potentials were: $(\mathrm{A})+0.60 \mathrm{~V}$, and (B) $-0.70 \mathrm{~V}$ vs. $\mathrm{Ag} / \mathrm{AgCl} / \mathrm{KCl}$ (saturated) reference electrode. Scan rate: $3 \mu \mathrm{m} \mathrm{s}^{-1}$. Tip diameter: $10 \mu \mathrm{m}$. Tip-substrate distance: $10 \mu \mathrm{m}$.

(A)

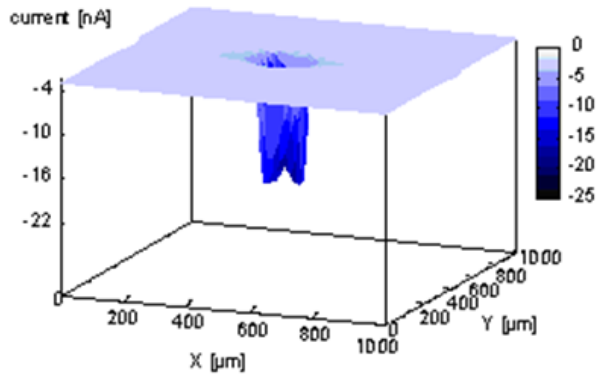

(B)

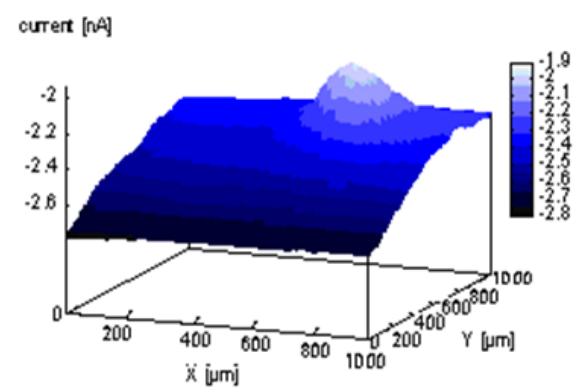

Figure 12:

SECM images of steel substrates painted by an epoxy-polyamine coating containing glass flakes as pigment after 7 hours immersion in: (A) $0.1 \mathrm{M} \mathrm{Na}_{2} \mathrm{SO}_{4}$, and (B) $0.1 \mathrm{M} \mathrm{Na}_{2} \mathrm{~B}_{4} \mathrm{O}_{7}$ solutions. The images represent $1000 \mu \mathrm{m} \times 1000 \mu \mathrm{m}$ in $X$ and $Y$ directions; scan rate: $30 \mu \mathrm{m} \mathrm{s}^{-1}$. Values of $Z$ axis: Current, nA. Redox mediator: oxygen. Tip potential: $-0.50 \mathrm{~V}$ vs. Ag/AgCl/KCl (saturated) reference electrode. Tip diameter: $10 \mu \mathrm{m}$. Tip-substrate distance: $15 \mu \mathrm{m}$. 